\title{
Die Jahresrechnung der Arztpraxis - Führungsinstrument oder «nur» Grundlage für die Steuererklärung?
}

Christian Flury

Korrespondenz:

Christian Flury, dipl. Wirtschaftsprüfer Blaser Treuhand AG FMH Treuhand Services Weissenbühlweg 26 CH-3007 Bern Tel. 0313701120 Fax. 0313714518

\section{Jahresrechnung im allgemeinen}

Ziel jeder Praxis ist es, das finanzielle Gleichgewicht durch geeignete Massnahmen zu erhalten. Es gilt, den Kapitalbedarf zu ermitteln und zu decken. Dies wird erreicht, indem die Zahlungsfähigkeit der Praxis jederzeit gesichert ist und eine Überschuldung (Schulden grösser als Eigenkapital) vermieden wird.

Die Buchführung mit daraus resultierender Jahresrechnung dient als Führungsinstrument. Der Jahresabschluss einer Praxis (Einzelfirma) besteht in der Regel aus der Bilanz, der Erfolgsrechnung sowie dem Kapitalnachweis. Die Bilanz am Bilanzstichtag (z. B. 31. Dezember), ist zu vergleichen mit einem Foto, also einer Momentaufnahme. Diese gibt Auskunft über das Vermögen (Aktiven) und Schulden (Passiven). Das erzielte Jahresergebnis ergibt sich aus der Differenz zwischen Anfangs- und Endbestand des Eigenkapitals, sagt aber wenig aus über das Zustandekommen dieser errechneten Zahl. Die Erfolgsrechnung (Gewinn- und Verlustrechnung) listet Einnahmen und Ausgaben des ganzen Jahres auf und ist zu vergleichen mit einem Film, dessen Ablauf uns nun genauestens über die Ursachen des Jahresergebnisses informiert, also eine Zeitraumrechnung. Mittels Analyse können wir genau prüfen, durch welche Einnahmen und Ausgaben das Jahresergebnis erzielt wurde. Wie wichtig die Analyse und das Errechnen von entsprechenden Kennzahlen sind, zeigt der Umstand, dass das Überleben einer Praxis und einer Unternehmung im allgemeinen von der Zahlungsfähigkeit abhängt. Eine ungenügende Ertragskraft beschränkt die geschäftlichen Entwicklungsmöglichkeiten, aber auch die private Existenz. So sollten in der Regel die Privatbezüge nicht grösser sein als das Jahresergebnis, da dies sonst die Eigenkapitalbasis einer Praxis schmälert, wobei aber gesagt werden muss, dass immer eine Gesamtbetrachtung anzuwenden ist, d.h., dass die Privat- wie Geschäftssituation miteinzubeziehen sind.

Die gesetzliche Buchführungspflicht nach Art. 957ff OR liefert ein absolutes Minimum an Vorschriften, mit denen zuverlässige Informatio- nen über die Vermögens- und Ertragslage bereitgestellt werden, um einen realistischen Einblick in die wirtschaftliche Situation der Praxis zu erhalten.

\section{Die Jahresrechnung als Führungsinstrument}

Als wichtige Aufgaben des Praxisinhabers erachten wir:

- Budgetierung der Ein- und Ausgaben;

- Soll-Ist-Vergleiche (Budget mit effektiven Zahlen vergleichen);

- Überwachung eines gesundes Verhältnisses zwischen Fremd- und Eigenkapital;

- Kostengünstige Fremdfinanzierung;

- Investitionspolitik;

- Mahnwesen Patientenrechnungen überwachen oder Outsourcing.

In der Regel befasst sich der Praxisinhaber (Einzelfirma) persönlich mit der finanziellen Führung seiner Arztpraxis, haftet er doch mit seinem ganzen Privatvermögen auch für die geschäftlichen Verbindlichkeiten.

Infolge Arbeitsüberlastung und auch familiärer Verpflichtungen delegiert er die Buchführung oder zumindest die Erstellung der Jahresrechnung oft an einen externen Berater. Die Führung der Finanzbuchhaltung im Betrieb hat den Vorteil, immer auf aktuelle Zahlen zugreifen zu können. Wichtig ist unseres Erachtens aber, dass die Person, die mit dieser Aufgabe beauftragt ist, ein Zahlenflair und Freude an dieser Arbeit hat, ansonsten es effizienter und schliesslich auch kostengünstiger ist, diese Aufgabe an einen Dritten zu delegieren.

Nach Vorliegen der Jahresrechnung kann das Zahlenmaterial ausgewertet werden, je schneller die Jahresrechnung vorliegt, desto besser und desto eher kann auf das neue Geschäftsjahr Einfluss genommen und allenfalls Kurskorrekturen vorgenommen werden. Bei der Analyse der Jahresrechnung handelt es sich allerdings um eine vergangenheitsorientierte Betrachtung, die dennoch wichtig ist, bildet sie doch die Grundlage für eine erfolgreiche Zukunft der Praxis. 
Unabhängig von der Grösse einer Unternehmung ist es unverzichtbar, Instrumente der Finanzplanung einzusetzen, sie aber der Grösse der Praxis anzupassen.

Nachstehend eine Auswahl von wichtigen Kennzahlen und Führungsinstrumenten:

- Bilanz- und Erfolgsanalyse im allgemeinen;

- Vorjahresvergleiche;

- kurzfristige Erfolgsrechnungen (Zwischenabschlüsse);

- Budgetkontrolle;

- Liquiditätskennzahlen errechnen;

- Cash-flow;

- Branchenvergleiche.

Es würde zu weit führen, diese Zahlen hier nun im einzelnen zu erläutern. Ihre FMH Treuhand Services Vertrauenspartner werden sich auf Ihre Anfrage hin gerne die Zeit nehmen, Ihnen diese näherzubringen.

In der Regel wird die Jahresrechnung mit Ihrem Treuhänder besprochen und durch diesen auch erläutert. Dies könnte auch der Anlass sein, die eine oder andere Kennzahl ausrechnen zu lassen und generell über die finanzielle Führung der Praxis ein Gespräch zu führen.

\section{Die Jahresrechnung als Grundlage für die} Steuererklärung bzw. Steuerbelastung

Nebst dem Führungsinstrument dient die Jahresrechnung auch der Erstellung der Steuererklärung, ist sie doch der Ausweis über den Erfolg des letzten Geschäftsjahres. Es handelt sich eigentlich um eine Art Lohnausweis, den jeder Arbeitnehmer von seinem Arbeitgeber erhält. Es ist also eine Zusammenstellung des Praxisinhabers über seine eigenen finanziellen Aktivitäten, ein Erfolgsausweis.

Die Steuern (öffentliche Abgaben) dienen dem Staat in erster Linie zur Deckung seiner Ausgaben. Sie werden aufgrund der rechtlich relevanten $\mathrm{Zu}$ gehörigkeit des Pflichtigen zum Gemeinwesen erhoben. Wenn wir von der Steuererklärung sprechen, so sprechen wir automatisch von den direkten Steuern, wie z. B. die Einkommens- und
Vermögenssteuern, die theoretisch nicht zur Überwälzung bestimmt sind und jeden treffen.

Nun zahlt bekanntlich niemand gerne zuviel Steuern. Klar ist aber auch, dass die Steuern ein nötiges Muss sind, damit der Staat seine vielfältigen Aufgaben im Auftrag seiner Bürger bewältigen kann. Steuern ja, aber nicht zuviel, ist die Devise einer steueroptimalen Abschlussgestaltung.

Hier einige Stichworte, die bei der Erstellung der Jahresrechnung mitzuberücksichtigen sind:

- Abschreibungspotential, allfällige Möglichkeit von Sofortabschreibungen, je nach Kanton;

- Wertberichtigungen auf Patientenguthaben (Delkredere);

- persönliche Beiträge des Praxisinhabers an die 2. Säule;

- Äufnung der Arbeitgeberbeitragsreserve;

- Abgrenzungen;

- Entschädigung für die Mitarbeit des/der Ehegatten/Ehegattin;

- Stichwort: Einkauf fehlende Beitragsjahre;

- Unterhaltskosten Liegenschaft;

- Möglichkeiten der 3. Säule (auch für den/die Ehegatten/Ehegattin).

Die Aufzählung ist natürlich nicht abschliessend. Sie soll vielmehr ein Anhaltspunkt sein, sich gelegentlich mit der Treuhandstelle zu unterhalten, ob die persönliche Jahresrechnung noch Steueroptimierungspotential hat. Durch eine legale optimale Abschlussgestaltung und weitere Instrumente der Vorsorge lässt sich viel an Steuern sparen. Zuviel bezahlte Steuern sind ein Ärgernis, das es zu vermeiden gilt.

\section{Führungsinstrument oder «nur» Grundlage für die Steuererklärung?}

Abschliessend kann festgehalten werden, dass die Eingangsfrage mit «sowohl als auch» beantwortet werden kann. Die obigen Erläuterungen haben gezeigt, wie wichtig die Jahresrechnung für die Analyse ist und dass sie tatsächlich ein Führungsinstrument darstellt, aber ebenso die Steuerlast beeinflusst. 